\title{
The number of deaths among infants under one year of age in England with pertussis: results of a capture/ recapture analysis for the period 2001 to 2011
}

A J van Hoek (albertjan.vanhoek@hpa.org.uk) ${ }^{1}$, H Campbell ${ }^{1}$, G Amirthalingam ${ }^{1}$, N Andrews ${ }^{2}$, E Miller ${ }^{1}$

1. Immunisation, Hepatitis and Blood Safety Department, Health Protection Agency, London, United Kingdom

2. Statistics Unit, Health Protection Services, Health Protection Agency, London, United Kingdom

Citation style for this article:

van Hoek AJ, Campbell H, Amirthalingam G, Andrews N, Miller E. The number of deaths among infants under one year of age in England with pertussis: results of a capture/recapture analysis for the period 2001 to 2011. Euro Surveill. 2013;18(9):pii=20414. Available online: http://www.eurosurveillance.org/ViewArticle.

aspx?Articleld $=20414$

Article submited on 14 September 2012 / published on 28 February 2013

Pertussis activity in England in 2012 was at its highest level for more than 12 years, leading to an increased number of deaths, especially among infants who were too young to be vaccinated. To support decision making on the introduction of maternal immunisation as an outbreak response measure to prevent these early deaths, we analysed reported deaths amongst infants of less than one year of age during the period from 2001 to 2011 with a capture/recapture analysis. We used log linear regression to allow for interactions. Reported deaths were obtained from the Hospital Episode Statistics for England, death registered by the Office of National Statistics and the enhanced surveillance of laboratory-confirmed pertussis conducted by the Health Protection Agency. There were a total of 48 deaths recorded; of these 41 had a disease onset before being fully protected by vaccination. Around half of these deaths (23) were recorded in all three datasets and 10 in only one. Due to the high coverage of the datasets the estimated number of deaths missed was small with 1.6 (95\% confidence interval (CI): $0.5-4.5)$ deaths. The total average incidence was 0.721 (95\% Cl: $0.705-0.763$ ) per 100,000 maternities. We concluded that under ascertainment of deaths from diagnosed pertussis cases is small.

\section{Introduction}

An increase in laboratory-confirmed cases of pertussis in England and Wales was observed from the third quarter of 2011, predominantly in adolescents and adults. This increase continued into 2012, leading to the highest recorded disease levels for more than 12 years [1], and extended into infants under three months who are at highest risk of severe complications, hospitalisation and death. This recent rise may partly be due to increased awareness amongst health professionals, leading to an improved ascertainment of cases. The reported rise in young infant cases, however, for whom ascertainment is more consistent through time, is likely to be indicative of a true increase in disease transmission. A national outbreak was declared in April 2012 [2] to initiate a nationally coordinated response to the ongoing increased pertussis activity, primarily to minimise further infant hospitalisations and deaths.

Increased pertussis activity has also been reported in other countries in recent years [3-5]. The reasons for these recent increases are not yet fully understood and a number of factors may be important. Improved ascertainment, the replacement of whole cell with acellular vaccines [6], genetic changes in Bordetella pertussis and waning protection have all been implicated $[7,8]$.

Increased mortality among infants who are too young to be fully vaccinated would be expected in association with a true rise in pertussis activity; in England and Wales this has been observed in 2012 with 13 deaths in infants with laboratory-confirmed pertussis reported by the end of November, exceeding the highest annual number of deaths in the last 10 years. As an outbreak control measure, the Departments of Health in the United Kingdom (UK), has recommended pertussis-containing vaccine for pregnant woman at 28 to 38 weeks gestation. Immunisation in pregnancy aims to induce passive immunity in the neonate through transplacental transfer of antibodies in the last trimester. It would also contribute to a cocooning effect by preventing disease in the mother, thereby indirectly protecting the infant against pertussis infection from birth.

To inform decision making regarding the introduction of such a programme, it is key to estimate the likely number of deaths to be prevented. The first step in this is to understand the completeness of ascertainment of pertussis-attributable deaths. It has previously been shown using the capture/recapture approach over the period from 1994 to 1999 that fatal pertussis cases were under-ascertained in England using the routine data sources, namely; laboratory-confirmed cases, deaths registered by the Office of National Statistics (ONS) and the Hospital Episode Statistics (HES) [9]. However over the last decade there might have been changes in recording and diagnostic practice. We therefore repeated the capture/recapture analysis for the period from 2001 to 2011. 


\section{Methods}

\section{Data sources}

Deaths due to pertussis were extracted from three different datasets: the Hospital Episode Statistics (HES), the Office of National Statistics (ONS) and the enhanced surveillance (ES) of laboratory-confirmed pertussis conducted by the Health Protection Agency (HPA) Immunisation, Hepatitis and Blood Safety Department (IHBSD). The ES is the only system that is based on laboratory-confirmed cases only; in the HES and ONS datasets cases may be clinically confirmed.

The HES dataset holds all records for hospitalised patients in England, including detailed information on diagnosis, operative procedures and discharge method. Patients with ICD10 code (the Tenth revision of the International Statistical Classification of Diseases and Related Health Problems: ICD-10) indicating whooping cough (codes beginning with $\mathrm{A}_{37}$ ) and an indication of death (recorded as method of discharge $=4$ and $/$ or discharge destination $=79$ ) were extracted.

The ONS mortality data is a record of all deaths in England and Wales. For each death there is a death certificate which records personal details and cause of death recorded by ICD-10 completed by the treating medical doctor or a coroner. Certificates recording the ICD-10 code beginning with ' $A 37$ ' in the field 'underlying cause of death' were extracted.

The ES system managed by the HPA IHBSD is based on all cases of pertussis that have been laboratory confirmed by the HPA Bordetella Reference Laboratory and/or reported to the HPA by a National Health Service (NHS) diagnostic laboratory in England and Wales. Confirmed cases are followed up by a surveillance questionnaire to the general practitioner to obtain information on vaccination status, hospitalisation and mortality. All deaths following a diagnosis of pertussis held on this database were extracted.

\section{Matching}

Cases in the three datasets were matched on sex, date of birth, name and date of death. Not all data items were available in all three datasets but each case could be unambiguously assigned to a matched or nonmatched status.

\section{Statistical analysis}

Due to limitations in the availability of HES data, the analysis was restricted to the years from 2001 to 2011 and only performed for deaths recorded in England. The number of maternities in England for those years was based on the recorded maternities in England and Wales [10] (2001-2011), assuming that 95\% of them were in England (based on the distribution in 2010). The analysis was performed for deaths in pertussis cases aged under one year, under six months and under 66 days. The $<66$ day cut-off excluded any infant potentially protected by at least one dose of pertussis-containing vaccine on the assumption that the earliest age at which the first dose is given is eight weeks (56 days) and that a minimum of 10 days is required to derive any vaccine-induced protection $(56+10=66$ days). Log linear Models were fitted to the data [11], and two-way

\section{TABLE 1}

Annual deaths due to pertussis in infants aged under one year and proportion captured by three surveillance systems, England, 2001-2011 ( $\mathrm{n}=48)$

\begin{tabular}{|c|c|c|c|c|}
\hline & Total & Office of National Statistics & Enhanced Surveillance ${ }^{a}$ & Hospital Episode Statistics \\
\hline 2001 & 8 & 5 & 6 & 5 \\
\hline 2002 & 4 & 4 & 1 & 2 \\
\hline 2004 & 3 & 3 & 3 & 3 \\
\hline 2005 & 7 & 5 & 7 & 4 \\
\hline 2006 & 5 & 1 & 4 & 3 \\
\hline 2007 & 4 & 4 & 4 & 4 \\
\hline 2008 & 6 & 6 & 6 & 5 \\
\hline 2009 & 2 & 2 & 2 & 2 \\
\hline 2010 & 1 & 1 & 1 & 1 \\
\hline 2011 & 5 & 3 & 5 & 3 \\
\hline Total & 48 & 36 & 39 & 34 \\
\hline
\end{tabular}

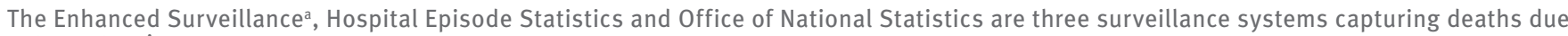
to pertussis.

\footnotetext{
a Coordinated by the Health Protection Agency.
} 
Reported pertussis deaths in England and notifications in England and Wales for persons aged less than one year per 100,000 maternities and 100,000 persons under one year-old respectively, 2001-2011

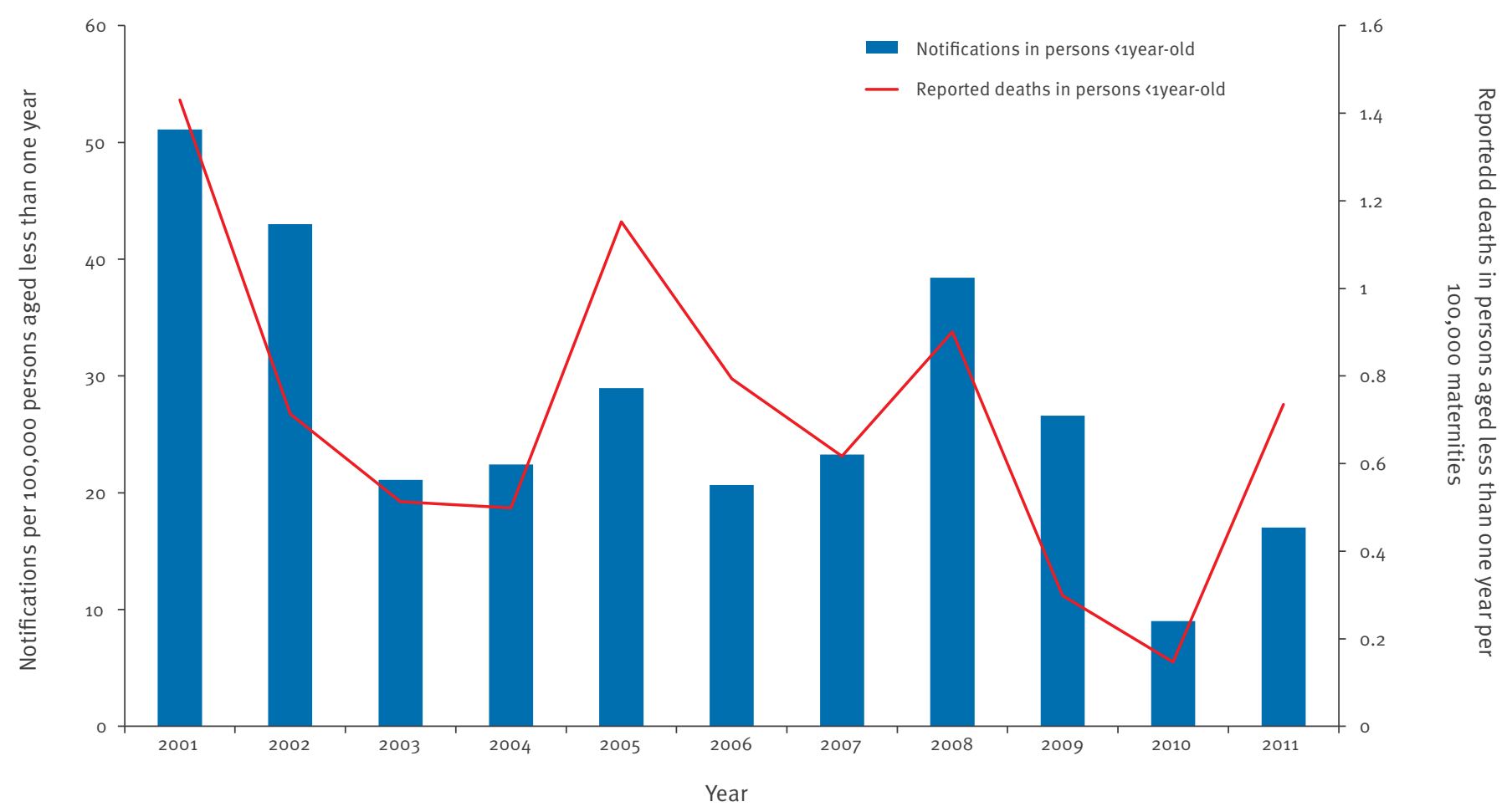

interactions between the terms were investigated. Confidence intervals $(95 \% \mathrm{Cl})$ were obtained by profile likelihood. All analyses were performed in R 2.14.1.

\section{Results}

There were 48 deaths in infants under one year of age identified in at least one dataset between 1 January 2001 and 31 December 2011; 23 (46\%) were in females. The number of deaths fluctuated between years, with the highest number in 2001 and the lowest in 2010 (Table 1). The annual mortality reflected the cyclical pattern in pertussis incidence in under one year-olds (Figure 1 and [12]). The distribution by age at death shows a clustering in the first two months of life (Figure 2) with 36 deaths occurring at this age; and of the 11 cases who died between two and six months of age, five had onset or specimen date before 66 days giving a total of 41 deaths $(36+5)$ in infants too young to be protected by a first dose of vaccine. Vaccination history was obtained for three of the remaining seven deaths; only one infant was vaccinated, nine days before specimen date.

The HPA ES recorded 39 deaths, ONS recorded 36 and 34 were recorded in HES, however almost half of the deaths were recorded in all three datasets (23/48) and only $21 \%$ were recorded in just one (6 in ES, and 2 in ENS and HES respectively) (Figure 3). Due to the low number of cases recorded in only one dataset the estimated number of missed deaths was low. The interaction between the HES and ONS databases was significant ( $p=0.03$, and 0.039 respectively) for all under one year-olds and all under six months of age; this interaction was therefore included for these two groups.

The total number deaths that were missed was estimated as 1.6 (95\% Cl: $0.5-4.5)$ for under one yearolds, 1.7 (95\% Cl: $0.5-4.7)$ for those under six months of age and $0.4(95 \% \mathrm{Cl}: 0.1-1.2)$ for those who died before reaching the age of 66 days (Table 2 ) over the full period. The estimated average risk of death including the missed cases was 0.721 per 100,000 maternities for all under one year-olds, 0.708 for all under six months of age and 0.601 for those under 66 days of age (including all those with known onset before 66 days). Expressed as an annual average number of deaths in England and Wales over the period 2001 to 2011 and based on the 716,040 maternities for England and Wales in 2011 [1], it is estimated that there were 5.2 (95\% Cl: $5.0-5.5)$ deaths in under one year-olds, 5.1 (95\% Cl: 4.9-5.4) deaths for those 66 months of age and 4.3 (95\% Cl: 4.3-4.4) for those aged under 66 days.

\section{Discussion}

Our study covered a period during which notified pertussis incidence was at an all time low, with sustained high vaccine coverage for the infant course, currently 
Number of reported pertussis deaths in infants aged less than one year, by age at death, England, 2001-2011 ( $\mathrm{n}=48$ )

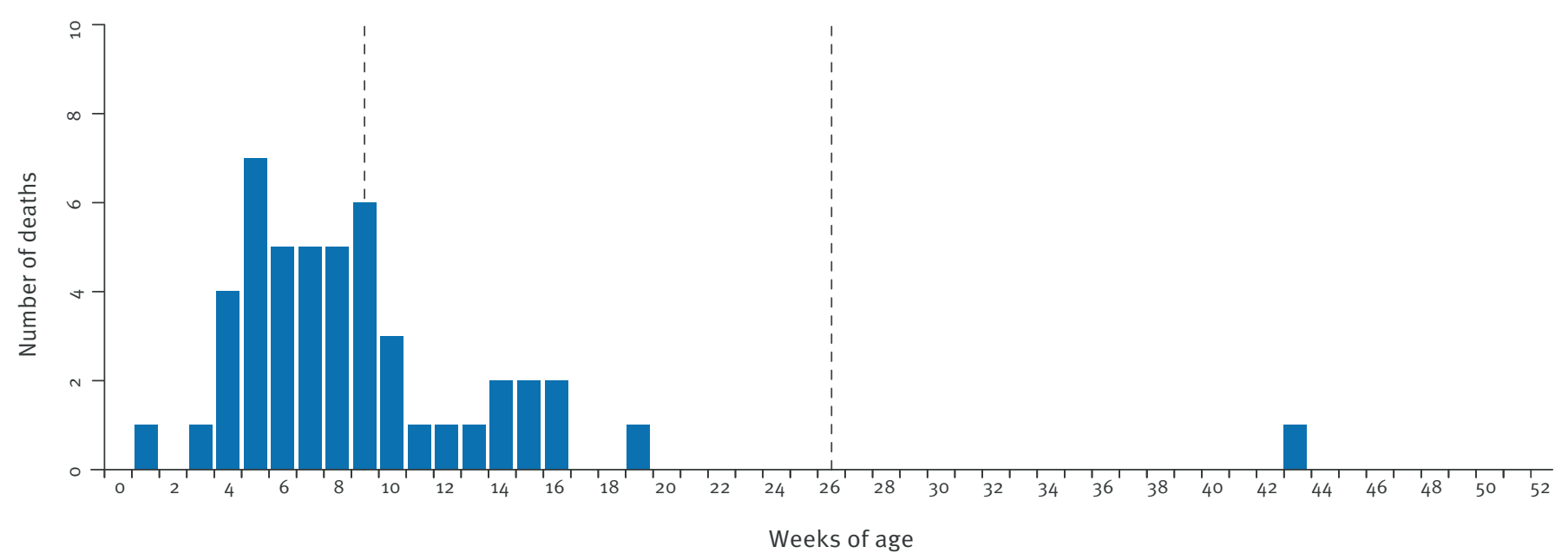

The dotted lines indicate the ages of 66 days and six months respectively.

The 66 day cut-off is the earliest time an infant can be potentially protected by at least one dose of pertussis-containing vaccine on the assumption that the earliest age at which the first dose is given is eight weeks ( 56 days) and that a minimum of 10 days is required to derive any vaccine-induced protection $(56+10=66$ days).

\section{FIGURE 3}

Venn diagram of the number of reported deaths due to pertussis in infants less than one year-old for three datasets obtained by three respective surveillance systems, England, 2001-2011 (n=48)

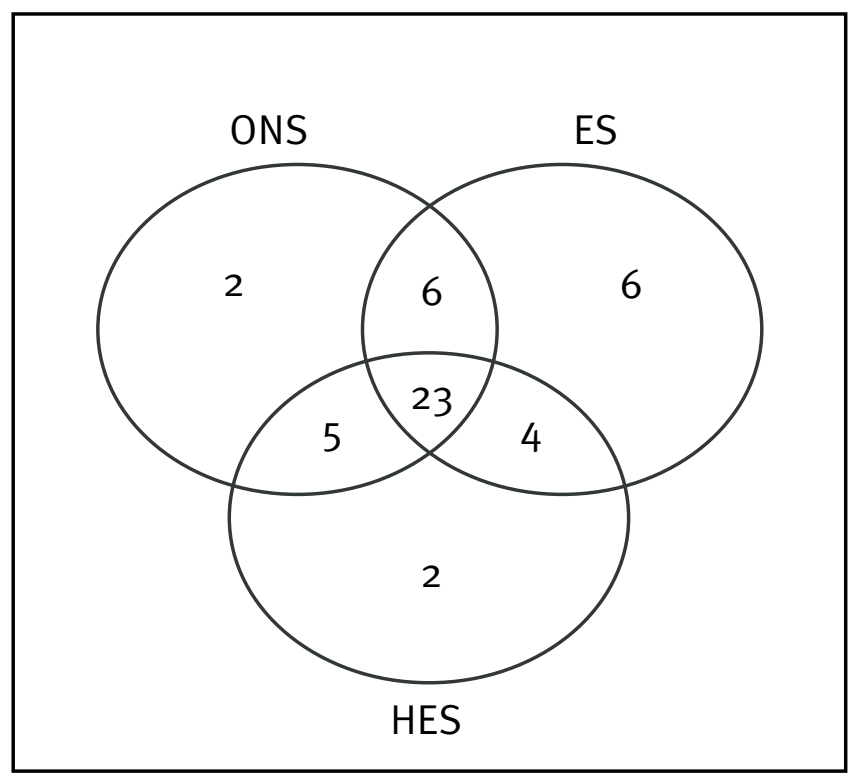

ES: Enhanced Surveillance coordinated by the Health Protection Agency; HES: Hospital Episode Statistics; ONS: Office of National Statistics.

The ES, HES and ONS are three surveillance systems capturing deaths due to pertussis. at $93 \%$ by first birthday, and a pre-school booster included in the programme since 2001. Yet, an average of five deaths a year due to pertussis were estimated in England and Wales, the majority in infants too young to have received protection from a first dose of vaccine. The annual number of deaths fluctuated depending on whether it was a peak year reflecting the vulnerability of very young unvaccinated infants to changes in pertussis transmission [12]. The highest annual number of deaths was in 2001 (8 cases) which has been exceeded in 2012 with 13 deaths reported by the end of November: a reflection of the large increase in pertussis transmission since the third quarter of 2011.

Our analysis shows that ascertainment of deaths in infants with diagnosed pertussis is fairly complete when using information from the three available data sources. However, there are limitations in using known fatal cases to estimate pertussis-attributable mortality. Inevitably, the analysis does not include deaths that are not diagnosed or registered as being related to pertussis. While this number cannot be estimated in our study, it was found that even when the infant has laboratory-confirmed disease the death certificate may not mention pertussis (10 of 39 laboratory-confirmed cases, Figure 3). This confirms an earlier study in which only two of seven infants with notified pertussis who died from the acute illness respiratory complications had pertussis recorded on the death certificate [13]. Pertussis has also been implicated in cases of sudden infant death syndrome, although the relationship remains speculative $[14,15]$. Of the 48 deaths in 


\begin{tabular}{|c|c|c|c|c|c|c|}
\hline Age at death & $\begin{array}{l}\text { Total } \\
\text { observed } \\
\text { deaths }\end{array}$ & $\begin{array}{l}\text { Estimated deaths } \\
\text { missed n }(95 \% \mathrm{Cl})\end{array}$ & $\begin{array}{c}\text { Total corrected } \\
\text { deaths n }(95 \% \mathrm{Cl})\end{array}$ & $\begin{array}{c}\text { Total deaths } \\
\text { observed } \%(95 \% \mathrm{Cl})\end{array}$ & $\begin{array}{l}\text { Interaction between } \\
\text { surveillance systems }\end{array}$ & $\begin{array}{c}\text { Estimated average } \\
\text { deaths per } 100,000 \\
\text { maternities } \mathrm{n}(95 \% \mathrm{Cl})\end{array}$ \\
\hline$<1$ year & 48 & $1.6(0.5-4.5)$ & $49.6(48.5-52.5)$ & $96.7(91.4-98.9)$ & ONS $^{\mathrm{a}}$ HES $p=0.035$ & $0.721(0.705-0.763)$ \\
\hline$<6$ months & 47 & $1.7(0.5-4.7)$ & $48.7(47.5-51.7)$ & $96.5(90.9-98.9)$ & ONS $^{\mathrm{a}}$ HES $p=0.039$ & $0.708(0.690-0.751)$ \\
\hline$<66$ days & $41^{\mathrm{a}}$ & $0.4(0.1-1.2)$ & $41.4(41.1-42.2)$ & $99.0(97.2-99.8)$ & None & $0.601(0.597-0.613)$ \\
\hline
\end{tabular}

HES: Hospital Episode Statistics; ONS: Office of National Statistics.

a Includes the five deaths for which cases had onset of disease before 66 days of age.

our study, nine had no laboratory confirmation and diagnosis was thus made on clinical grounds. It is possible that some of these cases may have been wrongly attributed to pertussis, resulting in an overestimate of mortality. However, on balance, given the lack of sensitivity of laboratory confirmation and the atypical presentation of pertussis in infants who may not develop the characteristic whoop and paroxysmal cough [16], an underestimate of mortality seems more likely.

We expressed infant mortality as deaths per 100,000 maternities rather than live births in order use the data in a cost-effectiveness analysis of a maternal pertussis immunisation policy. Maternities differ from live births in that vaccinating pregnant women will provide additional benefit in the event of the birth of a twin/ triplet but have no benefit in the case of a stillbirth. Expression of deaths per 100,000 maternities is thus a more accurate measure of the infant mortality potentially preventable by a maternal immunisation policy.

A capture/recapture analysis was previously performed for England using the same three datasets for the years 1994 to 1999 [9]. This suggested that only $70 \%$ (33 of an estimated 46) of deaths were ascertained, in contrast to our estimate of $97 \%$. The previous estimate included a year for which there was no information from HES, as hospital data was only available up to March 1998. Excluding this year changes the results with only 0.91 deaths missed of a total of 22. This gives an estimated $96 \%$ of all known deaths observed, similar to the percentage estimated from our study. The absolute number of observed deaths of 33 in six years in the earlier study gives only a slightly higher annual average (5.5) compared with our estimate of 4.4 (48 infant deaths observed in 11 years) despite a considerably higher notification rate in the 1990 [12]. This suggests that laboratory confirmation or clinical recognition of pertussis may have improved in recent years.

In conclusion, under ascertainment of known pertussis deaths is small when using our three national data sources though undiagnosed fatal cases may still be occurring. More deaths occur from pertussis than from any other disease covered by the current routine childhood immunisation programme. Mortality in vulnerable infants too young to be protected by vaccination reflects the overall population transmission of pertussis and interventions to protect young infants from exposure or development of disease if exposed are needed if pertussis mortality is to be reduced. The only intervention currently available that can protect infants from birth in all settings is immunisation in pregnancy. Such an approach can potentially protect the infant by the transfer of maternal antibodies and also reduce the likelihood of exposure to an infectious mother.

\section{Acknowledgements}

We thank Sonia Ribeiro for provision of the enhanced surveillance data on laboratory confirmed cases and Julia Stowe for provision of the HES data extracts. Albert Jan Van Hoek was supported by a grant from the DH Research and Development Directorate grant number 039/0031. The HES data is re-used with permission of the Health and Social Care Information Centre. All rights reserved. 


\section{References}

1. Health Protection Agency (HPA). Incidence of laboratoryconfirmed pertussis, by total case-patients and age group in England and Wales, 1998 - 2012. London: HPA. [Accessed 4 Sep 2012]. Available from: http://www.hpa.org.uk/webc/ HPAwebFile/HPAweb_C/1317135453069

2. Health Protection Agency (HPA). Confirmed pertussis in England and Wales continues to increase. 2012. London: HPA. [Accessed 4 Sep 2012]. Available at: http://www.hpa.org.uk/ hpr/archives/2012/news1512.htm\#prtsss

3. Fisman DN, Tang P, Hauck T, Richardson S, Drews SI, Low DE, et al. Pertussis resurgence in Toronto, Canada: a populationbased study including test-incidence feedback modeling. BMC public health. 2011;11:694. http://dx.doi.org/10.1186/14712458-11-694 PMid:21899765 PMCid:3189138

4. Somerville RL, Grant CC, Scragg RK, Thomas MG. Hospitalisations due to pertussis in New Zealand in the preimmunisation and mass immunisation eras. J Paediatr Child health. 2007;43(3):147-53. http://dx.doi.org/10.1111/j.14401754.2007.01034.X PMid:17316188

5. Tanaka M, Vitek CR, Pascual FB, Bisgard KM, Tate JE, Murphy TV. Trends in pertussis among infants in the United States, 1980-1999. JAMA. 2003;290(22):2968-75. http://dx.doi. org/10.1001/jama.290.22.2968 PMid:14665658

6. Sheridan S, Ware R, Grimwood K, Lambert S. Number and order of whole cell pertussis vaccines in infancy and disease protection. JAMA. 2012;308(5):454-6. http://dx.doi. org/10.1001/jama.2012.6364 PMid:22851107

7. Berbers GA, de Greeff SC, Mooi FR. Improving pertussis vaccination. Hum vaccine. 2009;5(7):497-503.

8. Crowcroft NS, Britto J. Whooping cough--a continuing problem. BMJ. 2002;324(7353):1537-8. http://dx.doi.org/10.1136/ bmj.324.7353.1537

9. Crowcroft NS, Andrews N, Rooney C, Brisson M, Miller E. Deaths from pertussis are underestimated in England. Arch Dis Child. 2002;86(5):336-8.

http://dx.doi.org/10.1136/adc.86.5.336

PMid:11970924 PMCid:1751114

10. Office National Statistics (ONS). Birth summary tables England and Wales 2011; table 1. London: ONS; 2012.

11. Cormack R. Log-linear models for capture-recapture. Biometrics. 1989;45:395-413. http://dx.doi. org/10.2307/2531485

12. Campbell H, Amirthalingam G, Andrews N, Fry NK, George RC, Harrison TG, et al. Accelerating control of pertussis in England and Wales. Emerg Infect Dis. 2012;18(1):38-47. http://dx.doi. org/10.3201/eid1801.110784 PMid:22260989 PMCid:3381681

13. Miller C, Fletcher WB. Severity of notified whooping cough. Br Med J. 1976;1(6002):117-9. http://dx.doi.org/10.1136/ bmj.1.6002.117

14. Heininger U, Stehr K, Schmidt-Schläpfer G, Penning R, Vock R, Kleemann $W$ et al. Bordetella pertussis infections and sudden unexpected deaths in children. Eur J Pediatr. 1996;155(7):551-3. http://dx.doi.org/10.1007/BF01957903 PMid:8831076

15. Nicoll A, Gardner A. Whooping cough and unrecognised postperinatal mortality. Arch Dis Child. 1988;63(1):41-7. http:// dx.doi.org/10.1136/adc.63.1.41

16. Ranganathan S, Tasker R, Booy R, Habibi P, Nadel S, Britto Pertussis is increasing in unimmunized infants: is a change in policy needed? Arch Dis Child. 1999;80(3):297-9. http://dx.doi. org/10.1136/adc.80.3.297 PMid:10325717

PMCid:1717870 